\title{
The Study of "Window-less" EDS Detector With Low Voltage FE-SEM
}

Yasuaki Yamamoto ${ }^{1}$, Hirobumi Morita ${ }^{2}$, Hirohisa Yamada ${ }^{3}$, Hideyuki Takahashi ${ }^{1}$, Masaru Takakura ${ }^{1}$, Naoki Kikuchi ${ }^{1}$, Takeshi Nokuo ${ }^{1}$, Natasha Erdman ${ }^{4}$

1. JEOL Ltd., 3-1-2 Musashino, Akishima, Tokyo, Japan

2. Oxford Instruments KK, IS Building, 3-32-42, Higashi-Shinagawa, Shinagawa-ku, Tokyo, Japan

3. National Institute of Technology, Nara College, Yamatokoriyama, Nara, Japan

4. JEOL USA Inc., Peabody, MA U.S.A.

Energy Dispersive X-ray Spectrometry (EDS) with an SEM is very popular for elemental analysis of bulk materials, and this method is called SEM/EDS. The element range capability of conventional EDS is from Be to U. However, in fact, it is not easy to analyze Be contained in a compound, because the film located in front of conventional EDS detector causes strong absorption of characteristic X-rays from light elements. This film is called the "window". The "Window" is mounted to keep the high vacuum condition of EDS detector. Therefore a window-less EDS detector is more suitable for EDS analysis of light elements.

On the other hand, there is a growing need as EDS mapping at low voltage for tens nm particles, with improvement of SEM performance. Generally, high electron probe current is needed to generate characteristic X-rays from a specimen for EDS analysis, and the diameter of electron beam becomes larger. However, the diameter of electron beam must be kept small to have high spatial resolution for EDS analysis, and also low voltage is needed for reducing interaction volume. For the reasons set forth above, detection solid angle of EDS detector should be designed as large as possible to efficiently detect small amounts of generated characteristic X-rays at low voltage and small electron probe current.

Recently, we have tested a new SEM/EDS system for EDS mapping of a Be-Cu-Co alloy and Pt nanoparticle catalysts. This system is a schottky FE-SEM, JSM-7800F Prime, in combination with a $100 \mathrm{~mm}^{2}$ SDD window-less EDS detector. This system can provide high spatial resolution EDS analysis at low voltage and analyze from Li to U. An electron beam deceleration system which is called GBSH (Gentle Beam for Super High resolution) mode is mounted on JSM-7800F Prime, which can reduce chromatic aberration effect especially at low voltage. GBSH mode can be combined with the EDS system. Fig1. shows a schematic view of GBSH mode. It can be apply a maximum $-5 \mathrm{kV}$ specimen bias in GBSH mode. Using GBSH mode a high spatial resolution is achieved even at extremely low voltage. The window-less EDS detector, Oxford Instruments X-Max Extreme, is designed to detect characteristic Xrays from light element without any absorption. The front part of this EDS detector is like an ellipse shape, and therefore can be inserted close to the specimen surface with no interference to the SEM. Therefore, detection efficiency for characteristic X-rays is improved dramatically at small probe current. Fig.2 shows the results of EDS mapping acquired by new the SEM/EDS system and conventional EDS, Oxford Instruments X-Max ${ }^{\mathrm{N}} 150 \mathrm{~mm}^{2}$ with "window", for a Be-Cu-Co alloy. Both measurement conditions are $3 \mathrm{kV}$ of incident voltage, 950pA of electron probe current, and $20 \mathrm{~min}$ of acquisition time. EDS mapping using the new system shows distribution of Be in the alloy, much more clearly than conventional EDS. There is concentration of Be at darker contrast area on SEM BE image. This agrees well with the result of SXES (Soft X-ray Emission Spectrometer) analysis by JEOL SS-94000SXES (Fig.3). Fig 4 shows result of EDS mapping for a $\mathrm{Pt}$ nano particle catalyst sample. Measurement condition is $5 \mathrm{kV}$ of incident voltage, $\mathrm{x} 500,000$ of magnification and $450 \mathrm{pA}$ of electron probe current. 
The distribution of Pt nano particles down to around 5nm size, is clearly visible in the EDS maps. These results demonstrate that new SEM/EDS system is suitable for the light element analysis and low voltage high spatial resolution analysis.

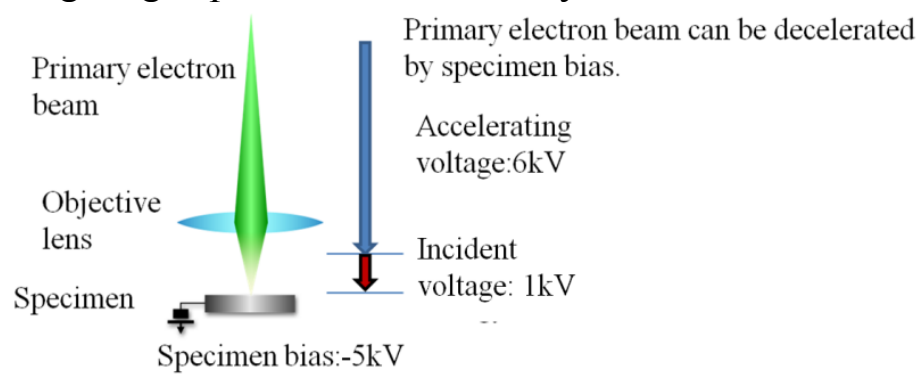

Figure 1. Method of GBSH mode
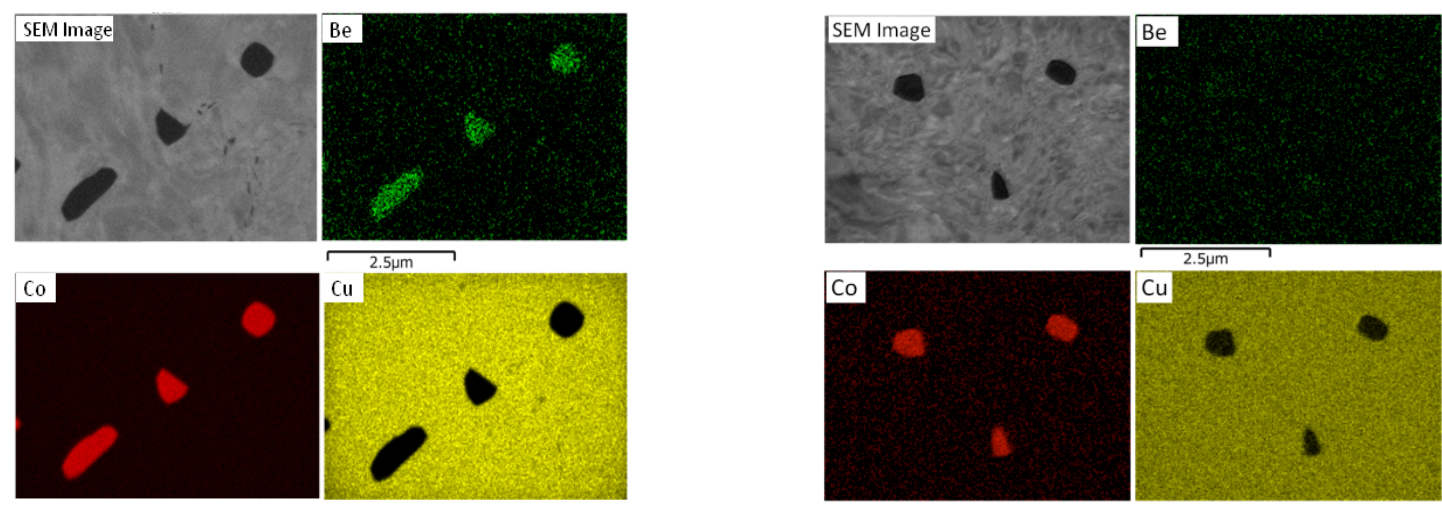

Figure 2. Comparison data of New SEM/EDS's mapping (left) and Conventional EDS's mapping (right) for a BeCuCo alloy

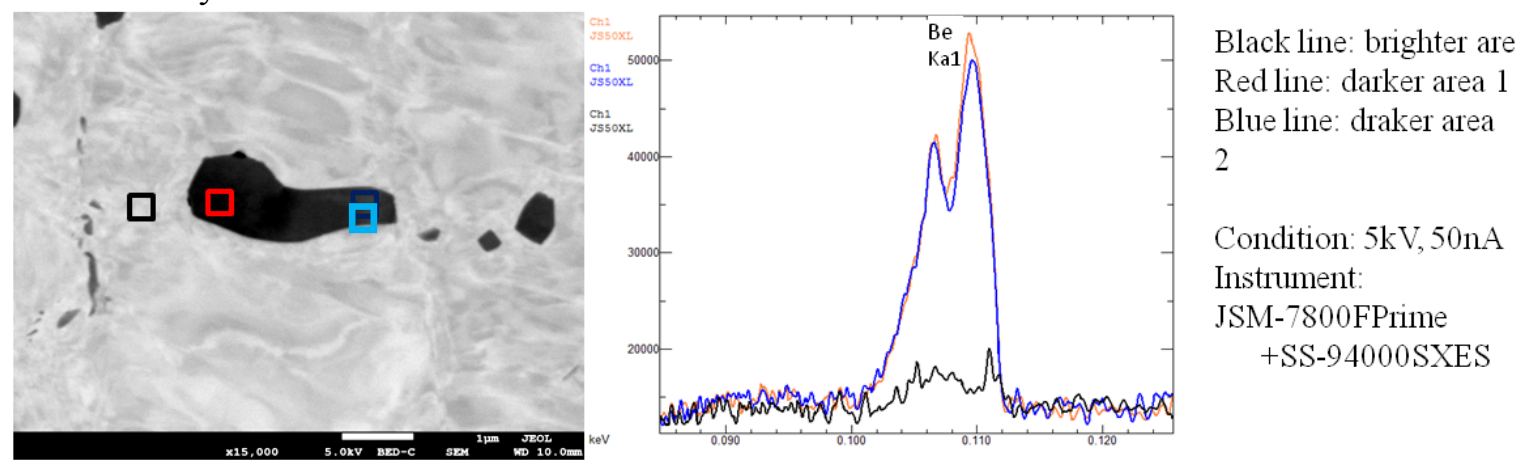

Figure 3. Point analysis of SXES for a BeCuCo alloy
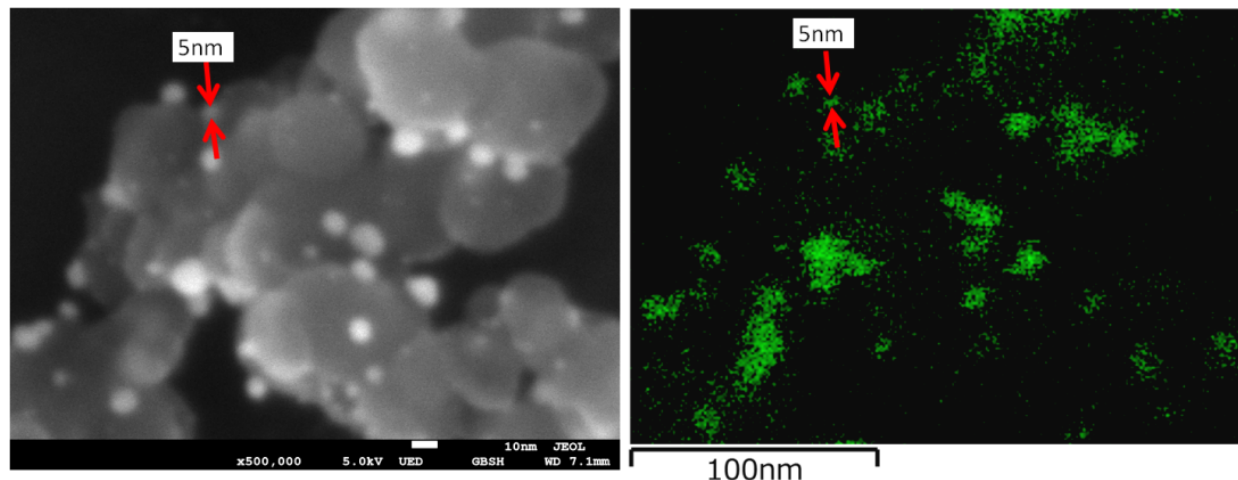

Figure 4. SEM image and EDS mapping by new SEM/EDS for a Pt nano particle catalyst 